\title{
Pertinence et pérennité de la restauration de chenaux latéraux: modèles issus de 15 ans de suivi sur le Rhône
}

\author{
Jérémie Riquier $^{1}$, Hervé Piégay ${ }^{1}$, Nicolas Lamouroux ${ }^{2}$ et Lise Vaudor ${ }^{1, *}$ \\ ${ }^{1}$ CNRS UMR 5600 EVS, université de Lyon, Lyon, France \\ 2 IRSTEA UR MALY, Villeurbanne, France
}

Reçu le 27 janvier 2017 / Accepté le 13 juin 2019

Résumé - Au cours des dernières décennies, de nombreux projets de restauration de chenaux latéraux ont été mis en œuvre, notamment pour améliorer le fonctionnement écologique de tronçons fluviaux de plaine fortement anthropisés. Néanmoins, les retours d'expérience concernant les ajustements morphologiques s'opérant après les travaux restent rares ou peu partagés, alors qu'ils semblent essentiels pour améliorer la conception technique de tels projets et évaluer leur durabilité. Cet article constitue une synthèse des résultats acquis depuis plus de 15 ans dans le cadre du suivi de 18 bras restaurés du Rhône. Les ajustements morphologiques enregistrés par les bras depuis leur restauration en termes de granulométrie et de sédimentation sont décrits. Puis, une estimation de leur durée de vie potentielle en tant qu'habitat aquatique est réalisée s'appuyant sur des modèles tendanciels. L'analyse de ces résultats suggère que la restauration des bras du Rhône constitue une solution à la fois pertinente et pérenne dans le contexte rhodanien. Nous démontrons également que les patrons granulométriques et les vitesses d'accumulation des fines peuvent être bien prédits à partir de descripteurs simples du fonctionnement hydrodynamique des bras. Ces modèles empiriques constituent des outils opérationnels pour guider la conception technique des futurs bras à restaurer du Rhône et potentiellement d'autres hydrosystèmes.

Mot clés : bras morts / restauration écologique / suivi / hydromorphologie / évaluation

\begin{abstract}
Relevance and sustainability of side channel restoration: models based on a 15-year monitoring along the Rhone River. Numerous projects of side channel restoration have been implemented during the last decades to improve the ecological functioning of highly regulated rivers. Feedbacks regarding the hydromorphological adjustment of restored channels are rare or not shared. Nevertheless, such information is crucial to improve the design of restoration measures and to assess their sustainability. In this paper, we report an overview of results derived from the monitoring of 18 restored side channels conducted over more than 15 years in the Rhône River. Firstly, hydromorphological adjustments of restored channels (bed material grain size longitudinal patterns, dynamics of fine sediment accumulation) are described. Then, an assessment of the potential persistence of channels as aquatic habitats is conducted using trend models. Our results suggest that side channel restoration can be a relevant and sustainable measure in the context of the Rhone River. We also demonstrate that grain size conditions and fine sediment accumulation rates can be successfully predicted using simple metrics reflecting the hydrodynamic functioning of side channels, that managers can target or modify: the frequency and magnitude (i.e. maximum shear stress) of upstream overflow events and the maximum intensity of backflow events (i.e. maximum backflow capacity). These empirical relationships are important operational tools for guiding future project design in the Rhone River and potentially elsewhere. They can be used to predict the effects and the sustainability of side channel restoration. They may reduce the uncertainty about the potential hydromorphological adjustments of side channels after restoration, and contribute to optimize the balance between local ecological gains and restoration costs.
\end{abstract}

Keywords: side channels / ecological restoration / monitoring / hydromorphology / evaluation

\footnotetext{
*Auteur correspondant : jeremie.riquier@gmail.com
} 


\section{Introduction}

Les chenaux latéraux (ou bras fluviaux) constituent des unités emblématiques des rivières de plaine, notamment lorsqu'elles ont conservé une certaine mobilité latérale. Les plaines alluviales peu domestiquées jouxtant les cours d'eau latéralement dynamiques se composent souvent d'un réseau de chenaux plus ou moins développés et interconnectés avec le cours principal. À l'échelle de la plaine alluviale, les bras fluviaux présentent souvent des stades successionnels diversifiés et disposent d'une forte hétérogénéité en termes de fonctionnement et d'habitat. Ils constituent in fine le support de communautés biologiques variées (Ward et al., 2002; Amoros et Bornette, 2002). Les grands cours d'eau de plaine, à l'instar du Rhône, comptent aujourd'hui parmi les écosystèmes les plus altérés et menacés à l'échelle mondiale. En tant qu'axes de développement majeur, ils ont fait l'objet d'aménagements (e.g. endiguements, barrages) à buts multiples (e.g. développement de la navigation, protection contre les inondations, production d'hydroélectricité) relativement lourds depuis le XIX ${ }^{\mathrm{e}}$ siècle. Ces infrastructures ont induit de profondes mutations à la fois physiques et biologiques, entraînant une diminution importante de la diversité structurelle et fonctionnelle des habitats et donc de leur richesse écologique (Petts et al., 1989; Ward et Stanford, 1995). En limitant certains processus clés de la dynamique fluviale, tels la divagation latérale ou encore le transit sédimentaire, ces aménagements ont engendré un affaiblissement notable de la capacité des cours d'eau à générer de nouveaux chenaux latéraux et à régénérer les plus anciens. Ces derniers tendent inéluctablement à s'atterrir (par comblement sédimentaire et/ou abaissement des lignes d'eau) et à disparaître des paysages fluviaux.

Dans de nombreux cas, le maintien des anciens chenaux latéraux au sein des corridors fluviaux ne peut plus être assuré sans la mise en œuvre de mesures curatives. Depuis une trentaine d'années, la prise de conscience de ces altérations a ouvert la voie à de nouvelles pratiques, visant à améliorer la qualité éco-hydromorphologique des hydrosystèmes fluviaux. Les chenaux latéraux font l'objet d'une attention croissante au sein des programmes de restauration des grands cours d'eau de plaine fortement anthropisés, en particulier pour améliorer leur fonctionnement écologique (Schiemer et al., 1999 [Danube en Autriche]; Baptist et al., 2004 [Waal et Rhin aux Pays-Bas]). Le suivi de ces opérations de restauration est fondamental, dans la mesure où il permet de fournir des informations essentielles pour la mise en œuvre de procédures de gestion adaptatives, pour guider la conception technique de futures opérations ou encore pour évaluer la durée de vie des écosystèmes restaurés qui est l'un des indicateurs du succès de ces opérations. Cependant, les retours d'expérience concernant l'ajustement hydromorphologique des bras restaurés sont encore rares dans la littérature scientifique internationale, alors que ces éléments de connaissance constituent un prérequis indispensable pour évaluer l'efficience des mesures de restauration. Dans ce contexte, l'objectif de cette contribution est de déterminer si le recreusement des chenaux latéraux du Rhône peut constituer une action de restauration à la fois pertinente, en termes de diversification des habitats, et pérenne. Autrement dit, il s'agit de confirmer le bien fondé d'un tel investissement.

\section{Restauration et suivi de chenaux latéraux du Rhône}

\subsection{Objectifs de la restauration}

La prise de conscience de la dégradation de l'hydrosystème rhodanien a conduit les décideurs à engager en 1998 un programme de restauration ciblant certaines sections courtcircuitées par les aménagements hydroélectriques du fleuve (les vieux-Rhône). Les opérations ont concerné l'amélioration des axes migrateurs et la restauration des habitats, avec deux mesures complémentaires :

- la modification raisonnée du régime des débits réservés qui doit permettre de redonner un caractère «vif et courant» aux vieux-Rhône;

- la remise en eau d'anciens chenaux latéraux du fleuve, à partir d'actions mécaniques, qui vise à augmenter la diversité d'habitats dans les plaines inondables et à favoriser les espèces caractéristiques de ces milieux.

Ce rajeunissement «artificiel» des bras repose sur la combinaison d'opérations de curage/dragage des alluvions fines et/ou grossières, s'associant parfois à la reconnexion totale (création de bras courant permanent) ou partielle (connexion permanente seulement par l'extrémité aval) des bras au chenal principal. Le rétablissement d'une diversité de modes d'alimentation en eau, de stades successionnels et de faciès hydromorphologiques a été recherché afin de maximiser la diversité des conditions d'habitat au sein et surtout entre les bras restaurés à l'échelle du tronçon fluvial (Amoros, 2001). En favorisant la diversité et l'hétérogénéité des habitats du lit mineur et du lit majeur, ces deux actions complémentaires visent à améliorer le fonctionnement écologique des tronçons fluviaux ciblés (Lamouroux et al., 2015). Entre 1999 et 2006, un total de 24 bras a été restauré sur 4 tronçons court-circuités : Pierre-Bénite en 1999, Chautagne en 2004, Belley en 2005 et Brégnier-Cordon en 2006. Dix-huit de ces bras font l'objet d'un suivi hydromorphologique, parmi d'autres thématiques (Lamouroux et al., 2015), dans le cadre plus général du programme d'accompagnement scientifique des opérations de restauration. Les lecteurs peuvent trouver des cartes de localisation des différents sites suivis dans Riquier (2015).

\subsection{Objectifs du suivi hydromorphologique des chenaux latéraux}

Le suivi hydromorphologique des bras a pour vocation de répondre à différents questionnements scientifiques répondant aux attentes opérationnelles. En effet, la stratégie de restauration des chenaux latéraux du Rhône, telle qu'elle est actuellement pratiquée, où l'on agit sur les symptômes et non sur les causes de la dégradation et où en quelque sorte «l'Homme» se substitue à la « nature», en recréant des formes que le cours d'eau n'est plus capable de créer lui-même, pose question. De nombreuses interrogations demeurent notamment quant à la pertinence et la pérennité de ces mesures sur le long terme, car elles peuvent apparaître comme «contre-nature». La notion de pertinence nous amène à questionner l'efficience des travaux réalisés en termes de diversification des conditions d'habitat entre les bras restaurés, à l'échelle des différents 
tronçons fluviaux ciblés. La notion de pérennité renvoie, quant à elle, à la durée de vie potentielle des bras restaurés en tant qu'habitat aquatique et donc à leur sensibilité aux processus d'atterrissement (i.e. comblement sédimentaire, changement de niveau de base) ou d'auto-entretien (i.e. décapages des dépôts). Ainsi, caractériser et comprendre les réponses hydrosédimentaires des chenaux latéraux restaurés et caractériser les processus qui rendent compte de leur variabilité dans le temps et dans l'espace constituent des enjeux importants à la fois sur le plan scientifique et opérationnel.

\section{Methodes}

\subsection{Protocole de suivi}

3.1.1 Granulométrie des dépôts et comblement des bras par les fines (caractérisation des réponses)

Après restauration, les bras restaurés ont fait l'objet de relevés in situ, réalisés en moyenne tous les deux ans, de manière à caractériser leur réponse morpho-sédimentaire. Nous disposons d'un état avant restauration pour 12 des 18 chenaux latéraux suivis et de 5 à 7 campagnes de mesures post-travaux offrant un recul temporel s'étendant de 7 à 15 années après restauration, selon les bras. Lors de ces campagnes, pour rendre compte de la distribution granulométrique des alluvions à l'intérieur des bras, cinq prélèvements équi-répartis sont réalisés longitudinalement dans l'axe du chenal avec une benne d'Eckmann. Ces échantillons sont par la suite analysés en laboratoire à l'aide d'un granulomètre laser. Tout échantillon dont la taille médiane estimée excède $2 \mathrm{~mm}$ est considéré comme matériel grossier, sans distinction de taille plus précise, et seuls les échantillons de fines sont prélevés. Ces mesures ont été utilisées dans le cadre de l'analyse de la diversité des conditions d'habitat et repose sur le postulat selon lequel les caractéristiques granulométriques des dépôts peuvent constituer un bon indicateur de la diversité des conditions fonctionnelles des bras. $\mathrm{Au}$ cours de ces campagnes, nous mesurons également la hauteur d'eau et l'épaisseur des dépôts de fines (fraction $<2 \mathrm{~mm}$ ) tous les $10 \mathrm{~m}$ de linéaire le long de l'axe médian de la zone en eau des bras. Ces relevés sont effectués à la pige ; un topofil est utilisé pour connaitre la distance parcourue depuis un point de référence fixe d'une campagne à l'autre. Ce second type de mesures est utilisé dans le cadre de l'analyse de la durée de vie des bras.

\subsubsection{Hydrodynamisme des écoulements de crue (caractérisation des contrôles)}

Afin de caractériser le régime d'écoulement en crue des bras, nous nous sommes appuyés pour chaque tronçon d'étude sur des chroniques de débit horaire et sur les relations hauteurdébit établies dans le chenal principal au droit de l'ensemble des bras étudiés et dans les bras connectés de manière permanente avec le chenal principal uniquement par l'aval. Des relevés topographiques des bouchons alluviaux amont de ces bras ont également été réalisés à l'aide d'un DGPS, pour déterminer le cheminement préférentiel des écoulements superficiels lors de leur submersion et les cotes altimétriques de débordement. L'ensemble de ces données nous ont permis d'estimer les paramètres hydrologiques et hydrauliques qui constituent les facteurs de contrôle clés des conditions sédimentaires observées dans les bras. Les trois variables retenues permettent de quantifier:

- la fréquence des débordements amont engendrant un fonctionnement lotique des bras (i.e. fréquence de connexion amont, exprimée en jours.an ${ }^{-1}$ );

- la force maximale s'exerçant sur le fond enregistrée par les bras depuis leur restauration en fonctionnement courant $(i$. $e$. contrainte de cisaillement maximale, exprimée en $\mathrm{N}$. $\mathrm{m}^{-2}$; il s'agit d'une valeur moyenne à l'échelle du bras);

- l'intensité du reflux dans le bras lorsque celui-ci se trouve en phase d'alimentation par retours d'eau depuis son extrémité aval (i.e. capacité maximale de reflux, exprimée en $\mathrm{m} . \mathrm{km}^{-1}$ ).

Cette métrique correspond au différentiel de hauteur d'eau mesuré entre le niveau observé juste avant la submersion du bouchon alluvial amont et celui observé pour le débit minimal du vieux-Rhône (débit réservé), rapporté à la longueur de la zone en eau permanente du bras.

\section{Résultats}

\subsection{Caractérisation de la diversité des conditions d'habitat (pertinence des actions)}

L'analyse des données de granulométrie des sédiments échantillonnés dans les bras (Fig. 1) a permis de mettre en évidence que la part spatiale (inter-bras) de la variance totale des conditions granulométriques observées après restauration est très forte $(81 \%)$. Ce résultat souligne que la diversité de ces conditions est bien plus importante entre les différents chenaux latéraux que celle résultant des trajectoires temporelles individuelles de chaque bras. Une analyse en composantes principales et des classifications ont permis d'identifier quatre principaux types de conditions granulométriques moyennes, de facto robustes et pour l'instant stables dans le temps (Fig. 1). Les chenaux de type 1 sont des bras à lit graveleux pouvant présenter des plaques sporadiques de sables, voire parfois de limons. Les bras de types 2 et 3 constituent des cas intermédiaires présentant des taux de recouvrement de leur fond par des dépôts de sédiments fins similaires. Les bras de type 2 enregistrent des dépôts plutôt sablo-limoneux, alors que ceux de type 3 s'inscrivent dans une gamme plutôt limonosableuse. Les bras de type 4 sont, quant à eux, totalement recouverts par des dépôts limoneux. La plupart des bras présentent des conditions granulométriques après travaux très proches de celles observées avant restauration. Ainsi, les travaux ont avant tout permis de rajeunir les formes sans pour autant modifier les processus contrôlant les modalités de sédimentation des bras.

\subsection{Estimation de la durée de vie des bras restaurés (pérennité des actions)}

Les bras présentent une large gamme de patrons de sédimentation (Fig. 2). L'ensemble des bras, à l'exception de MOLO, enregistrent des processus de décapage lors d'évènements de crue relativement fréquents (compris entre la crue biennale et quinquennale, selon le tronçon fluvial considéré). Ceci souligne que ces bras possèdent une certaine capacité d'auto-entretien. 


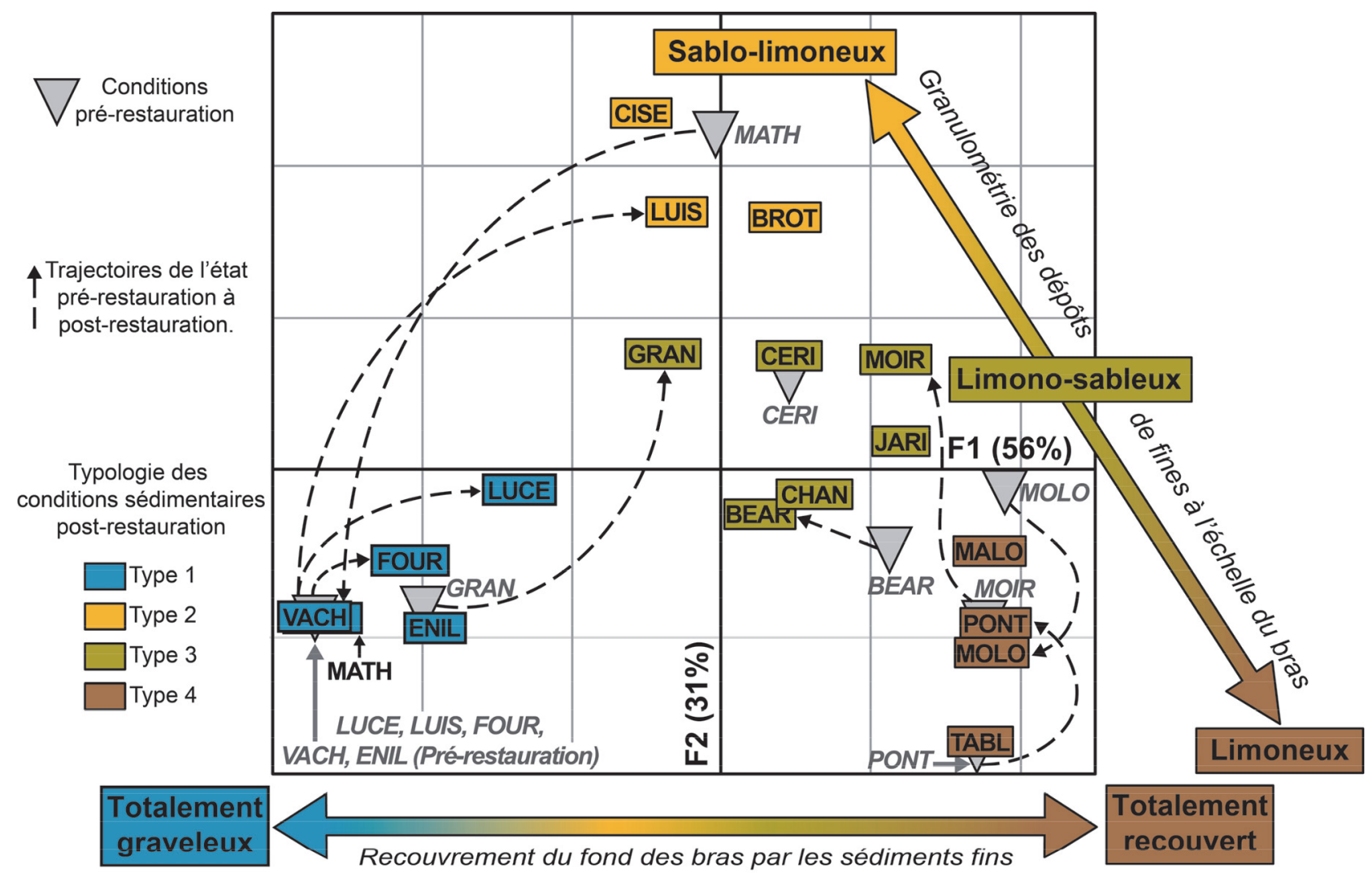

Fig. 1. Analyse de la diversité des conditions granulométriques de surface moyennes mesurées dans les bras étudiés (modifié et adapté d'après Riquier et al., 2015). Plan factoriel simplifié obtenu à partir d'une analyse en composantes principales inter-bras. Les bras sont ordonnés principalement selon les caractéristiques granulométriques des dépôts sédimentaires et le taux de recouvrement de leur fond par les sédiments fins. Les types de conditions ont été déterminés à partir d'une classification ascendante hiérarchique réalisée sur les deux premières composantes de l'analyse. La position des bras après restauration (code à 4 lettres) correspond à une condition moyenne reposant sur l'ensemble des observés disponibles. Les observations disponibles réalisées avant restauration ont été projetées sur le plan factoriel, permettant ainsi d'apprécier l'effet des travaux réalisés sur les conditions granulométriques observées ( $c f$. ibid. pour détails méthodologiques).

Pour aller plus loin dans cette analyse, nous avons exploré la significativité des relations observées à partir de tests de permutation (Fig. 2). Sur les 16 bras pouvant être intégrés dans cette analyse, l'évolution de l'épaisseur moyenne des sédiments fins est significativement liée au temps pour 10 d'entre eux. En d'autres termes, ces chenaux tendent à accumuler de plus en plus de sédiments fins au fur et à mesure que le temps s'écoule. Les processus de décapage dans ces bras peuvent les rajeunir épisodiquement, sans pour autant assurer leur auto-entretien, leur capacité de rajeunissement n'étant jusqu'à présent pas assez importante pour contrecarrer les processus de sédimentation. Les 6 autres chenaux présentent, quant à eux, un effet non significatif $\mathrm{du}$ temps, soulignant qu'ils sont a priori capables d'autoentretenir leur stade aquatique sur le long terme.

Pour explorer la relation entre $\overline{E p}$. l'épaisseur moyenne des sédiments fins accumulés et $T$ le temps écoulé depuis la restauration des bras, nous avons utilisé deux formes d'ajustement potentielles comme indiqué dans les équations suivantes :

$$
\begin{gathered}
\overline{E p .}=\alpha \cdot T^{(1-\beta)}, \\
\overline{E p .}=\alpha \cdot(1-\exp [-\beta \cdot T]),
\end{gathered}
$$

où $\alpha$ reflète la propension du bras à accumuler des sédiments fins, $\beta$ est un paramètre de forme qui reflète la décroissance (ou non) de l'accumulation des sédiments fins au cours du temps. Deux types d'approche ont été testés. D'une part, nous avons construit des modèles «classiques » où les paramètres alpha et beta sont calculés individuellement pour chacun des bras (i.e. modèles à effets fixes). D'autre part, nous avons utilisé des modèles mixtes à effets aléatoires. Ici, le paramètre bêta est représenté par une valeur unique et fixe pour l'ensemble des bras, représentant le comportement global des bras en termes de ralentissement (ou non) des vitesses de comblement au cours du temps. Le paramètre alpha est quant à lui dit aléatoire, dans la mesure où il est calculé pour chaque bras. Au final, on dispose donc de quatre tendances potentielles pour chaque bras.

À partir de ces modèles, nous avons pu fournir une première estimation des gammes de durée de vie potentielle des bras (Tab. 1). Quatre situations ont été identifiées pour les 16 bras analysés : 6 ont une durée de vie a priori infinie, car ils sont capables de s'auto-entretenir ; 4 ont une longévité potentielle supérieure à un siècle ; 3 ont des durées de vie que l'on qualifiera d'intermédiaire, s'étendant d'environ 3 à 9 décennies, c'est pour ces bras que l'incertitude est la plus forte (cette forte variation est liée au déclin ou non des vitesses de sédimentation au cours du temps) ; enfin, 3 


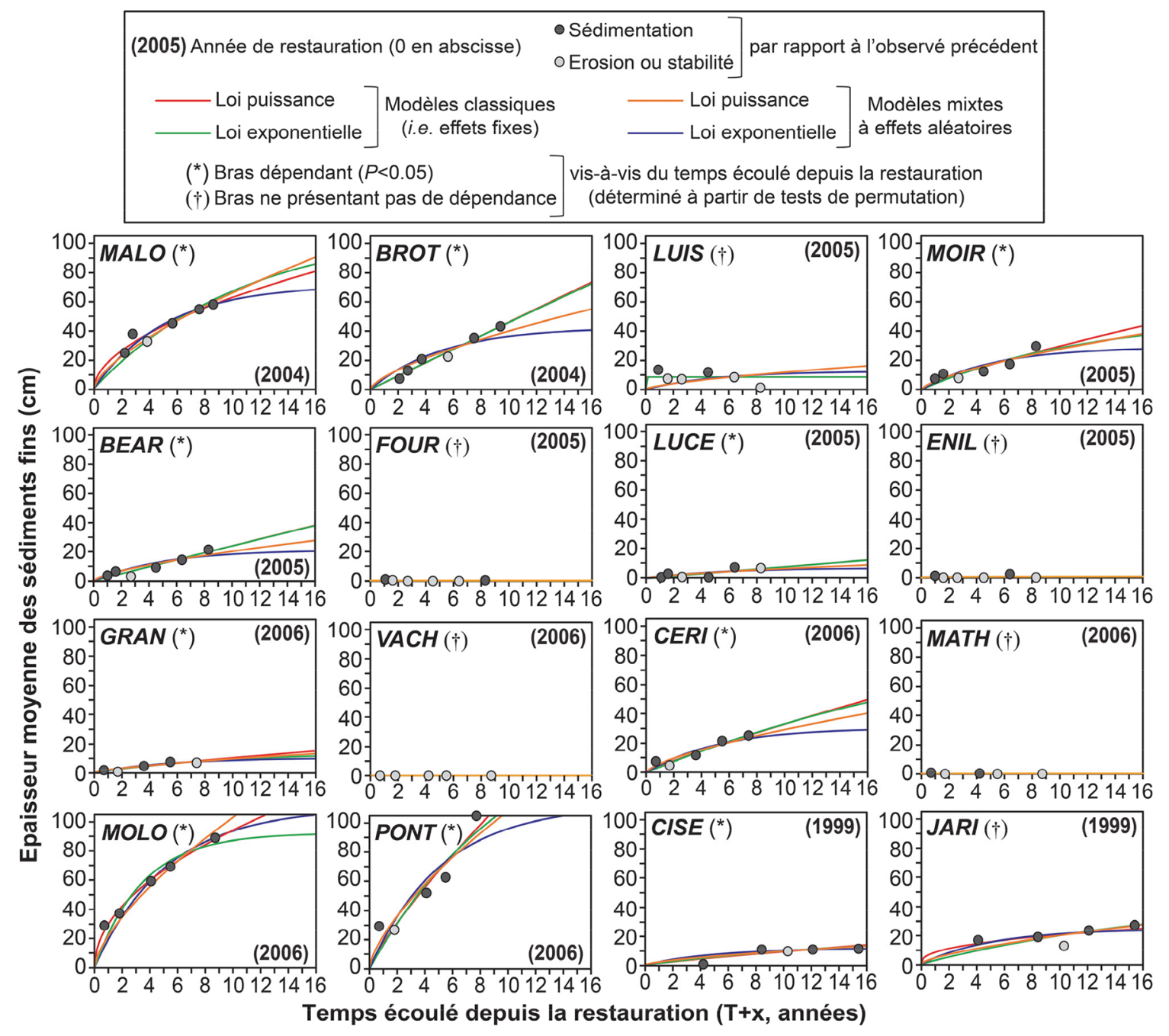

Fig. 2. Évolution du comblement sédimentaire des bras étudiés après restauration (modifié et adapté d'après Riquier et al., 2017).

Tableau 1. Estimation de la durée de vie potentielle des bras restaurés en tant qu'habitats aquatiques (modifié et adapté d'après Riquier et al., 2017).

\begin{tabular}{lllllllll}
\hline & MALO & BROT & LUIS & MOIR & BEAR & FOUR & LUCE & ENIL \\
Durée de vie (années) & $35-47$ & $34-99$ & Auto-entretien & $51-90$ & $35-93$ & Auto-entretien & $>100$ & Auto-entretien \\
& GRAN & VACH & CERI & MATH & MOLO & PONT & CISE & JARI \\
& $>100$ & Auto-entretien & $>100$ & Auto-entretien & $21-32$ & $17-22$ & $>100$ & Auto-entretien \\
\hline
\end{tabular}

Pour estimer la durée de vie des bras en tant qu'habitat aquatique, nous avons extrapolé les tendances modélisées (Fig. 2), afin d'obtenir différents scénarios d'évolution potentielles des épaisseurs moyennes de fines. Nous avons ensuite calculé le temps nécessaire pour que l'épaisseur sédimentaire moyenne soit équivalente à la hauteur d'eau moyenne des bras mesurées juste après restauration. Au final, seules les tendances les plus réalistes ont été retenues pour chaque bras.

${ }^{a} \mathrm{Ce}$ bras semble avoir atteint un certain équilibre métastable. Cependant, rien ne nous permet d'affirmer que cet état va perdurer.

autres bras ont une durée de vie courte, comprise entre $c a .2$ et 5 décennies. Il convient toutefois de garder à l'esprit que ces estimations sont des projections des tendances actuelles. À ce titre, nous ne savons pas encore comment les bras réagiront à des crues moins fréquentes que celles qu'ils ont déjà subies (entre $\mathrm{Q}_{5}$ et $\mathrm{Q}_{20}$ selon le tronçon considéré). D'autre part, nos modèles considèrent que l'atterrissement des bras est seulement contrôlé par leur comblement par les fines. D'autres facteurs peuvent affecter les hauteurs d'eau mesurées dans les bras, comme par exemple une évolution du niveau de base dans les bras (e.g. incision du chenal principal), ou un engravement pour les bras présentant des écoulements très compétents. 

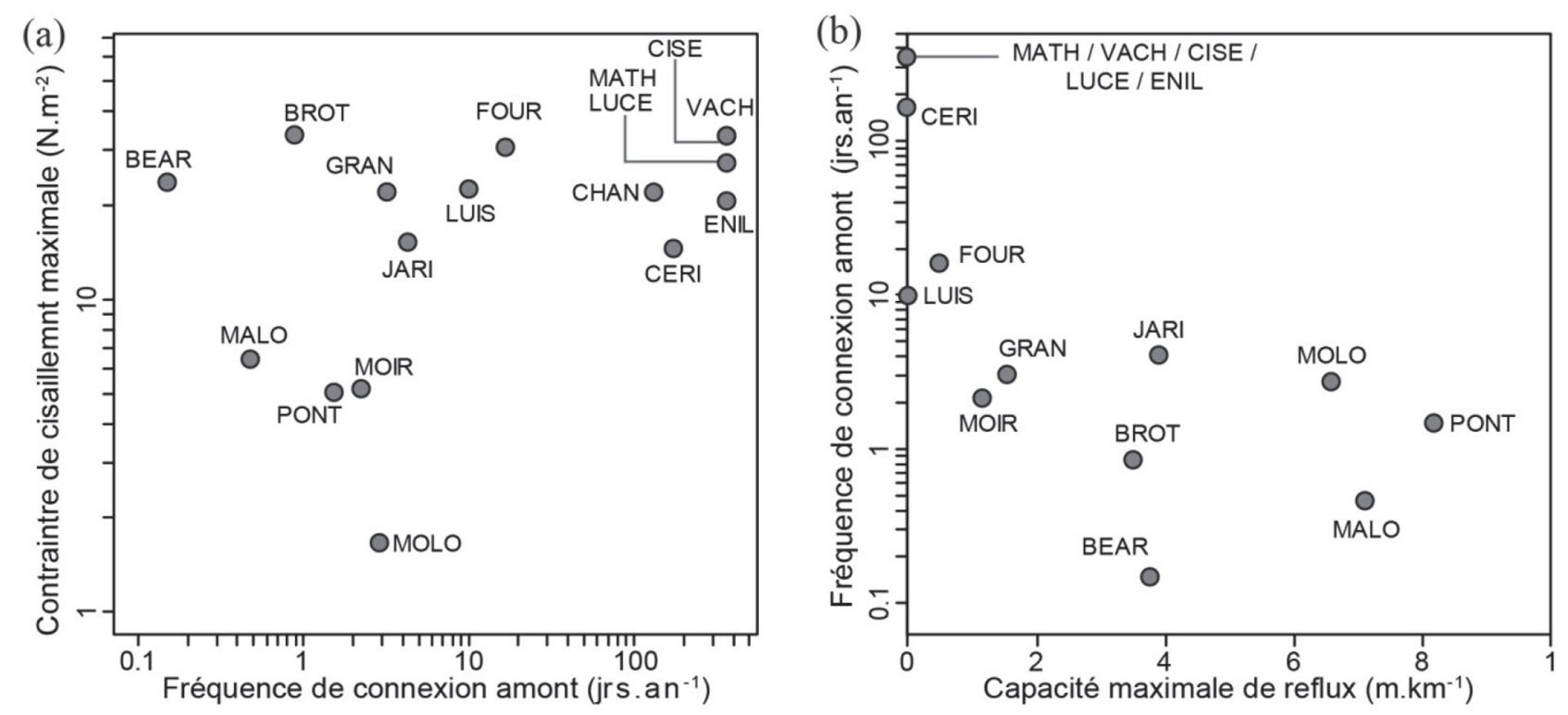

Fig. 3. Caractérisation de l'hydrodynamisme des bras en crue (modifiée et adaptée d'après Riquier, 2015).

\subsection{Prédiction a priori du type fonctionnel et de la propension des bras à sédimenter}

Les bras restaurés présentent une diversité de fonctionnements hydrodynamiques. La figure $3 \mathrm{a}$ montre ainsi qu'ils enregistrent, plus ou moins fréquemment, un écoulement courant. $\mathrm{Au}$ cours de ces épisodes de connexion amont, l'intensité des écoulements varie de manière conséquente d'un bras à l'autre, indépendamment de la fréquence de cette connexion. La figure $3 b$ permet de mettre en évidence ainsi deux principaux types de fonctionnement: d'une part, des bras fréquemment connectés à l'amont $\left(>10\right.$ jours.an $\left.{ }^{-1}\right)$ avec une capacité maximale de reflux nulle ou très faible et d'autre part, des bras peu fréquemment connectés $\left(<10\right.$ jours.an $\left.{ }^{-1}\right)$ avec des capacités de reflux très variées s'étendant de 1 à $8 \mathrm{~m} . \mathrm{km}^{-1}$.

Les variables permettant de caractériser le régime d'écoulement des bras en crue (Fig. 3) ont alors été utilisées pour tester notre capacité à prédire les ajustements hydromorphologiques s'opérant après leur restauration (Fig. 4). Les conditions granulométriques moyennes observées en surface dans les bras après restauration (ainsi que les types associés) (Fig. 4a) sont bien prédites à partir de ces descripteurs de l'hydrodynamisme de crue des bras, tout comme l'est également la propension des bras à accumuler des sédiments fins (ainsi que leur durée de vie potentielle dans une certaine mesure) (Fig. 4b).

Les modèles ont été construits à partir de régressions linéaires multiples. Les équations établies sont indiquées pour chaque exemple. Les types de conditions granulométriques moyennes observés en surface après restauration (Fig. 1) et les gammes de durée de vie potentielle des bras restaurés (Tab. 1 et Sect. 4.2) sont également représentés. En ce qui concerne la prédiction de l'accumulation de fines dans les bras, nous avons retenu les valeurs du paramètre alpha issues de la forme puissance du modèle mixte à effet aléatoire utilisant une loi puissance (Fig. 2). Cette approche nous permet de comparer les bras sur une base commune étant donné que dans ce cas le paramètre beta est fixe. L'ajustement selon la loi puissance a été retenu puisqu'il est plus performant (erreur quadratique moyenne) que celui utilisant la forme exponentielle. Les lecteurs peuvent trouver des éléments d'interprétation supplémentaires dans Riquier et al. (2019).

\section{Conclusions et perspectives}

L'évaluation de la pertinence des actions de restauration en termes de diversification des conditions d'habitat repose sur la granulométrie des dépôts de surface prise comme indicateur. Quatre principaux types fonctionnels ont ainsi été mis en évidence. Ceux-ci sont, jusqu'à présent, pérennes dans le temps. Nous avons également démontré que ces types de bras sont régis par les conditions hydrodynamiques observées lors des crues (Fig. 4a). Ces travaux ont donc bien confirmé qu'il existe une diversification des conditions hydrauliques et sédimentaires des bras restaurés, diversification qui se maintient au cours du temps. Ces éléments de conclusion sont corroborés par les résultats acquis par hydrobiologistes sur les bras restaurés des tronçons de Belley et de BrégnierCordon, qui démontrent que la restauration a permis d'augmenter la diversité bêta des peuplements de macroinvertébrés (Castella et al., 2015). La stratégie mise en œuvre dans le cadre de la restauration des chenaux latéraux du Rhône a donc bien permis de restaurer des bras disposant d'une diversité de stades et de dynamiques successionnelles. Elle semble donc constituer une solution de gestion pertinente des plaines rhodaniennes.

Les modèles tendanciels élaborés démontrent que les bras restaurés présentent des durées de vie potentielles variées, qui sont pour la plupart en accord avec les objectifs initiaux des opérateurs. Ces durées de vie s'étendent d'une vingtaine d'années, pour les bras les plus prompts à s'atterrir, à plusieurs siècles pour les bras présentant les plus fortes capacités d'autoentretien. Néanmoins, nous devons encore observer comment les bras réagiront à des évènements de crue moins fréquents que ceux qui ont été enregistrés jusqu'à présent (compris entre la crue biennale et quinquennale, selon le tronçon fluvial 
(a)

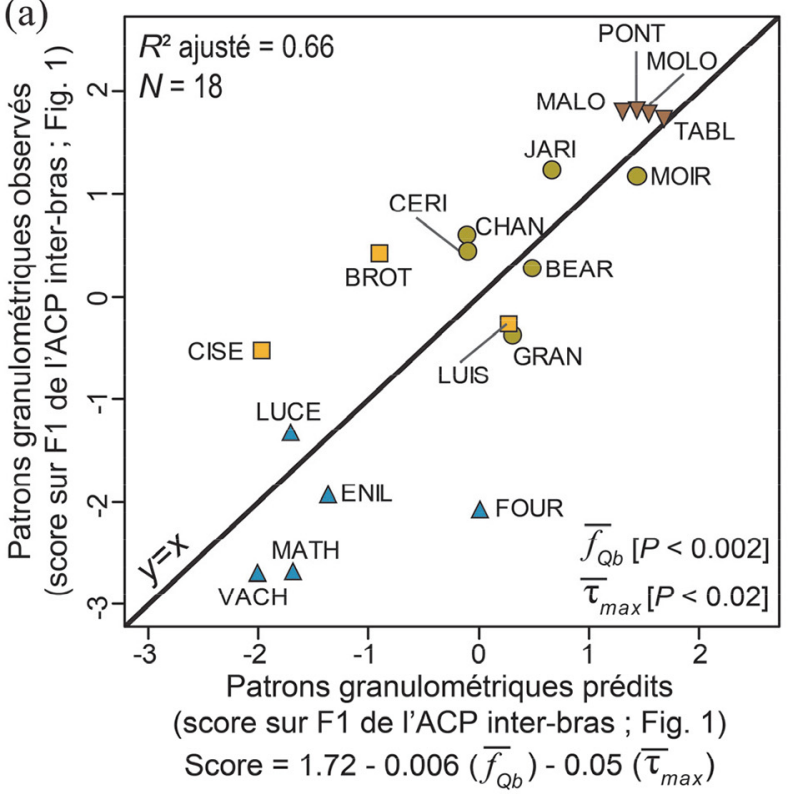

\begin{tabular}{|c|c|c|c|}
\hline $\begin{array}{c}\text { Typologie des conditions granulométriques } d \\
\begin{array}{ccc}\text { प Type } 1 & \square \text { Type } 2 & \text { OType } 3 \quad \nabla \text { Ty }\end{array}\end{array}$ & $\begin{array}{l}\text { e surface } \\
\text { pe } 4\end{array}$ & $\overline{f_{Q b}}$ & Fréquence de connexion amont \\
\hline $\begin{array}{l}\text { Durée de vie potentielle } \\
0 \text { Infinie } \bigcirc>100 \text { ans } 0 \text { ca. } 3 \text { à } 9 \text { décennies }\end{array}$ & - ca. 2 à 5 décennies & $\begin{array}{c}\bar{\tau}_{\max } \\
C R_{\max }\end{array}$ & $\begin{array}{l}\text { Contrainte de cisaillement maximale } \\
\text { Capacité de reflux maximale }\end{array}$ \\
\hline
\end{tabular}

(b)

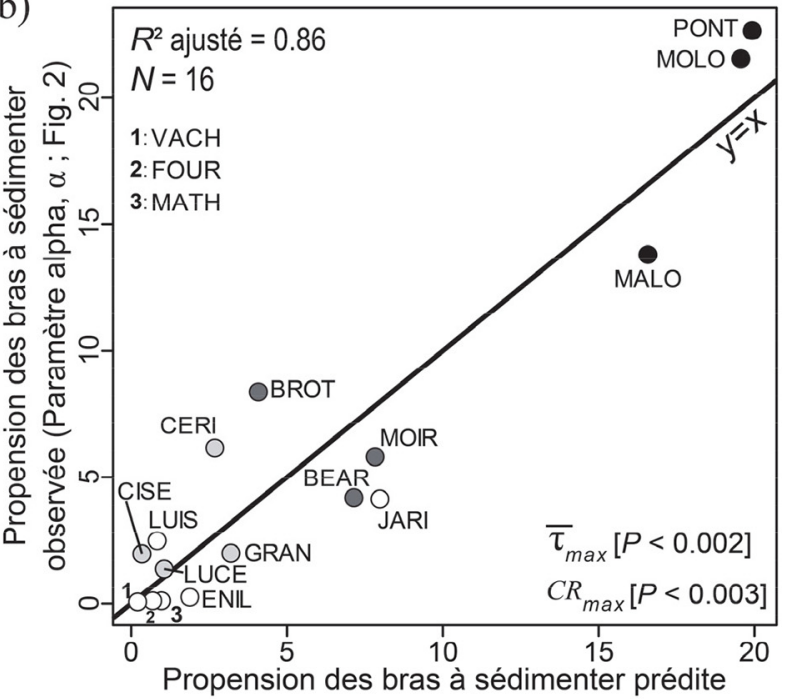

$\begin{aligned} & \text { (Paramètre alpha, } \alpha ; \text { Fig. } 2) \\ \alpha= & 12.6-3.5 \log \left(\bar{\tau}_{\max }\right)+1.6\left(C R_{\text {max }}\right)\end{aligned}$

Fig. 4. Exemple de relations empiriques permettant de prédire (a) les conditions granulométriques moyennes post-restauration et (b) la propension des bras à accumuler des sédiments fins à partir de leurs caractéristiques d'écoulement en crue (Fig. 3) [modifiée et adaptée d'après Riquier et al. (2015) et Riquier et al. (2017)].

considéré). À ce jour, nous avons considéré l'accumulation de sédiments fins comme le seul facteur contrôlant l'atterrissement des bras. Cependant, les niveaux d'eau observés dans les bras et de fait leur durée de vie peuvent également être affectés par d'autres facteurs, tels des changements de niveau de base imputables à des ajustements locaux de la géométrie du lit mineur (i.e. incision du lit, migration de radiers). Ce type de phénomène reste toutefois mineur sur le Rhône, étant donné que sur les 18 bras étudiés ici, seuls 2 présentent de tels ajustements (Riquier, 2015). Ainsi, d'un point de vue général, agir sur les formes semble constituer dans le contexte rhodanien une mesure pérenne. Cela est possible car la géométrie du lit mineur est souvent figée, le transport solide est faible à nul et les concentrations en matières en suspension sont peu élevées, comparativement à d'autres hydrosystèmes. Avec la mise en ouvre actuelle d'un schéma de réélargissement des marges fluviales et la réintroduction prévue d'une charge de fond potentiellement mobile, ce contexte va sans doute changer et la question de la pérennité de certains bras devra alors être mieux évaluée. La nouvelle géométrie devrait en effet avoir des effets en termes de connectivité hydrologique des bras en crue ou de niveau d'eau lors des écoulements en débit réservé.

Les relations statistiques établies (Fig. 4) démontrent qu'il existe un lien entre l'hydrodynamisme des écoulements de crue dans les bras restaurés, les conditions d'habitat physique et la durée de vie potentielle des bras. L'intérêt des variables de contrôle utilisées réside dans le fait qu'elles sont, au moins en partie, dépendantes de la géométrie des bras (e.g. cote de débordement amont ou conditions de pente), sur laquelle il est possible d'intervenir lors des travaux. Ainsi, il est maintenant possible d'évaluer a priori l'effet de la modification de la géométrie des bras restaurés sur l'ajustement hydrosédimentaire des bras après restauration. Le cadre typologique et les modèles statistiques produits constituent des outils opérationnels pertinents, qui vont permettre de mieux encadrer la conception technique des futures actions de restauration sur le Rhône et potentiellement ailleurs. Ces outils peuvent, en outre, permettre de réduire les incertitudes quant à la réponse écohydromorphologique potentielle des bras restaurés ou encore de maximiser les gains écologiques locaux et donc d'optimiser les futurs investissements financiers dans ce type d'opération. La transférabilité de ces modèles à d'autres hydrosystèmes sera testée dans les années à venir.

Remerciements. Ce travail a été réalisé dans le cadre d'une thèse soutenue à l'université Lumière Lyon 2 et financée dans le cadre du programme de recherche «RhônEco». Ce dernier bénéficie du soutien technique et financier de nombreux partenaires (Compagnie nationale du Rhône, Agence de l'eau Rhône-Méditerranée-Corse, régions Auvergne-Rhône-Alpes et Provence-Alpes-Côte d'Azur, Électricité de France, Union Européenne via le Fond européen de développement économique régional et de multiples collectivités rhodaniennes). Nous remercions vivement l'ensemble des personnes ayant participé à la collecte des données sur le terrain et à la réflexion scientifique et plus spécifiquement Jean-Michel Olivier, Emmanuel Castella et Monika Šulc Michalková.; 


\section{Références}

Amoros C. 2001. The concept of habitat diversity between and within ecosystems applied to river side-arm restoration. Environ Manage 28(6): 805-817.

Amoros C, Bornette G. 2002. Connectivity and biocomplexity in waterbodies riverine floodplains. Freshwater Biol 47(4): 761-776.

Baptist MJ, Penning WE, Duel H, Smits AJ, Geerling GW, Van der Lee GE, Van Alphen JS. 2004. Assessment of the effects of cyclic floodplain rejuvenation on flood levels and biodiversity along the Rhine River. River Res Appl 20(3): 285-297.

Castella E, Béguin O, Besacier-Monbertrand AL, Hug Peter DH, Lamouroux N, Mayor Siméant H, McCrae D, Olivier JM, Paillex A. 2015. Realised and predicted changes in the invertebrate benthos after restoration of connectivity to the floodplain of a large river. Freshwater Biol 60(6): 1131-1146.

Lamouroux N, Gore JA, Lepori F, Statzner B. 2015. The ecological restoration of large rivers needs science-based, predictive tools meeting public expectations: an overview of the Rhône project. Freshwater Biol 60(6): 1069-1084.

Petts GE, Möller H, Roux AL, eds. 1989. Historical change of large alluvial rivers: Western Europe. John Wiley \& Sons Ltd.
Riquier J. 2015. Réponses hydrosédimentaires de chenaux latéraux restaurés du Rhône français. Structures spatiales et dynamiques temporelles des patrons et des processus, pérennité et recommandations opérationnelles. Thèse de doctorat, Université Lumière Lyon 2, $293 \mathrm{p}$.

Riquier J, Piégay H, Šulc Michalková M. 2015. Hydromorphological conditions in 18 restored floodplain channels of a large river: linking patterns to processes. Freshwater Biol 60(6): 1085-1103.

Riquier J, Piégay H, Lamouroux N, Vaudor L. 2017. Are restored side channels sustainable aquatic habitat features? Predicting the potential persistence of side channels as aquatic habitats based on their fine sedimentation dynamics. Geomorphology 295: 507-528.

Schiemer F, Baumgartner C, Tockner K. 1999. Restoration of floodplain rivers: the 'Danube restoration project'. Regulated Rivers Res Manage 15(1): 231-244.

Ward JV, Stanford JA. 1995. Ecological connectivity in alluvial river ecosystems and its disruption by flow regulation. Regulated Rivers Res Manage 11(1): 105-119.

Ward JV, Tockner K, Arscott DB, Claret C. 2002. Riverine landscape diversity. Freshwater Biol 47(4): 517-539.

Citation de l'article : Riquier J, Piégay H, Lamouroux N, Vaudor L. 2019. Pertinence et pérennité de la restauration de chenaux latéraux : modèles issus de 15 ans de suivi sur le Rhône. La Houille Blanche 2: 101-108 